\title{
Phylogeography and historical demography of the Lusitanian snail Elona quimperiana reveal survival in unexpected separate glacial refugia
}

\author{
Aude Vialatte*, Annie Guiller, Alain Bellido and Luc Madec
}

Address: UMR CNRS ECOBIO, Université de Rennes 1, Campus de Beaulieu, 263 av. Gal Leclerc, CS 74205, 35042 Rennes Cedex, France Email: Aude Vialatte* - aude.vialatte@univ-rennes1.fr; Annie Guiller - annie.guiller@univ-rennes1.fr; Alain Bellido - alain.bellido@univrennes1.fr; Luc Madec - luc.madec@univ-rennes1.fr

* Corresponding author

Published: 19 December 2008

BMC Evolutionary Biology 2008, 8:339 doi: I0.1 I86/I47|-2| 48-8-339
Received: 9 June 2008

Accepted: 19 December 2008

This article is available from: http://www.biomedcentral.com//47I-2/48/8/339

(c) 2008 Vialatte et al; licensee BioMed Central Ltd.

This is an Open Access article distributed under the terms of the Creative Commons Attribution License (http://creativecommons.org/licenses/by/2.0), which permits unrestricted use, distribution, and reproduction in any medium, provided the original work is properly cited.

\begin{abstract}
Background: Present day distributions of Palearctic taxa in northern latitudes mainly result from populations having survived in local patches during the Late Pleistocene and/or from recolonizing populations from southern temperate refugia. If well-studied Mediterranean and eastern European refugia are widely accepted, some recent biogeographical assumptions still remain unclear, such as the occurrence of multiple glacial refugia in lberia and cryptic refugia in northern Europe during the last glaciations. The Lusitanian snail Elona quimperiana has a remarkably disjunct distribution, limited to northwestern France (Brittany), northwestern Spain and the Basque Country. By describing the phylogeographical structure of this species across its entire range, the present study attempts to identify refugia and subsequent recolonization routes.
\end{abstract}

Results: Results based on $16 \mathrm{~S}$ and $\mathrm{COI}$ gene sequences showed that the low genetic diversity observed in the Brittany populations should be associated with a recent demographic expansion. By contrast, populations from Spain exhibit several differentiated lineages and are characterized by demographic equilibrium, while the Basque populations are the only ones harboring typical distinct haplotypes. The center of the star-like networks of both gene sequences is occupied by a common ancestral-like haplotype found in Brittany and Spain, which might have originated from the middle of Northern Spain (i.e. Asturias, eastern Lugo and western Cantabria). Estimates of the divergence time between the Spain-Brittany and Basque lineages strongly suggest that $E$. quimperiana survived the Pleistocene glaciations in distinct refugia on the Iberian Peninsula, one of which is situated in Picos de Europa, and the other in the Basque Country. The occurrence of a northern refugium in France cannot be rejected as of yet.

Conclusion: Present results confirm the Iberian origin of the land snail E. quimperiana and strongly support the emerging phylogeographic hypothesis of multiple refugia in lberia during the last glaciations. The scenario of a spatial expansion of $E$. quimperiana from an lberian refuge located in Asturias to northern areas provides the most probable explanation for the present distribution of this land snail. By harboring distinct haplotypes, the Basque Country populations appear to be of great importance in terms of potential adaptation, long term persistence and hence, the conservation of $E$. quimperiana. 


\section{Background}

Geographically structured populations are the result of historical and/or contemporary demography. Some of them may experience either little or no genetic contact for long periods of time, due to physical, ecological or geographical barriers (e.g. [1-6]). More recent and rapid events, such as extinction, introduction or fragmentation, often linked to human activities, may also produce spatially partitioned populations (e.g. $[7,8])$.

For European species, the Quaternary glaciations are a major historical factor shaping the patterns of spatial population structure [9-13]. During these periods, the ice sheet induced the preservation of many species, but only in isolated refugia, resulting in population divergence via genetic drift and local selection [14]. The commonly recognized refugia in Western Europe during the Quaternary glaciations are on the Iberian and the Italian peninsulas $[10,14,15]$. It has been suggested that the northern regions of Europe were post-glacially colonized, generally from these refuges $[10,14]$. Recent phylogeographic and biogeographic studies have uncovered multiple refugia in Iberia (see [16] for a review). However, the number, location and habitat composition of these 'refugia in refugia' still remain poorly known. Scenarios other than the northern expansion from southern refugia have also been proposed. On one hand, cold-tolerant species, such as Mustela erminea [17], could have naturally expanded their repartition range during the Quaternary glaciations. On the other hand, the presence of additional putative refugia has been advanced to explain the distribution of poor cold-hardy species $[18,19]$. The hypothesis of local, but relatively recent and short-lived refugia much further north of the Mediterranean refugia is concordant with the discontinuity of the Pleistocene ice sheet in this region [20]. These temperate refugia are particularly recognized to explain the survival of some communities, such as freshwater fish [21]. The principal biome of unglaciated northern Europe during the late Pleistocene cold stages was the steppe tundra, i.e. a treeless vegetation type [22]. The presence of small isolated populations of trees in buffered local microclimates is also suspected in northwestern Europe during the Pleistocene; but, except for one controversial site in Belgium [18], no temperate forest refugia for animal species have actually been precisely localized in this part of Europe. Therefore, contemporary demographic factors are more likely to explain well-separated populations of poor cold-hardy species in Western Europe, such as human introductions (e.g. [23]), or the recent extinction of intermediate populations [24].

The land snail Elona quimperiana (Gastropoda, Xanthonychidae [25]) is a western Palearctic Helicoidea species with a highly disjunct distribution limited to Brittany in France (its name comes form the city of Quimper) and Northern
Spain (Fig. 1, [26]). It is a so-called Lusitanian species, i.e. a species that typically has a disjunct distribution in Iberia and southwest Ireland, with either no or highly disjunct populations in the regions between both areas (i.e. England and France) $[27,28]$. Elona quimperiana therefore provides an excellent opportunity to shed more light on European historical biogeography. This species lives in temperate and humid deciduous forests, where it feeds on mycelia found on rotten, dead stumps (principally oak). Occasionally, it is coprophagous and necrophagous [29]. Like many other terrestrial gastropods, E. quimperiana has a relatively limited dispersal capacity and probably survived during the Quaternary glaciations through significant fluctuations in its distribution area, just as its deciduous forest habitat did [30]. The present study is based on phylogeographical analyses that are highly successful in discriminating past and present demographic factors that were likely to have influenced the distribution of populations. The aims are to (i) describe the phylogeographical structure of E. quimperiana throughout its entire range, and (ii) identify the refugia and the subsequent recolonization routes.

\section{Results \\ Phylogenetic analysis}

The length of $16 \mathrm{~S}$ rDNA sequences ranged from 479 to $481 \mathrm{bp}$, with a total of 19 substitutions, including two indels at positions 123 and 420, while the length of the COI sequences was $683 \mathrm{bp}$, with a total of 47 substitutions. Eleven haplotypes were identified among the 54 snails analyzed for the 16S gene: eight from Spain, three from Brittany, and one from the Basque Country. Brittany had only one haplotype (haplotype 1) in common with Spain; this haplotype was the most frequent one, since 30 individuals shared it. Twenty-three haplotypes were obtained for the COI gene among the 81 individuals analyzed: eleven from Spain, six from Brittany (including one in common with Spain) and seven from the Basque Country. The most frequent haplotype (haplotype A), shared by more the half of the specimens, was also the only one common to both Brittany and Spain.

The networks based on the 16S rDNA and COI sequences exhibited convergent results. The 16S network showed two haplogroups (Hg1 and Hg11; Fig 2). Hg1 comprised 10 haplotypes (46 individuals) that were scattered throughout Spain and Brittany. The most frequent haplotype in this group (H1; 30 individuals) is distributed throughout all of Brittany (except at BZH3 Pont Calleck) and occurs in the Spanish provinces of Lugo, Asturias and western Cantabria (sites Sp2, Sp3, Sp4 and Sp5; Fig 3). All of the other $\mathrm{Hg} 1$ haplotypes are represented by either a single or few specimens. Haplotypes 2 and 3 are specific to Brittany and were only found at BZH2 (Montagnes Noires) and BZH3 (Pont Calleck). In Spain, a total of 


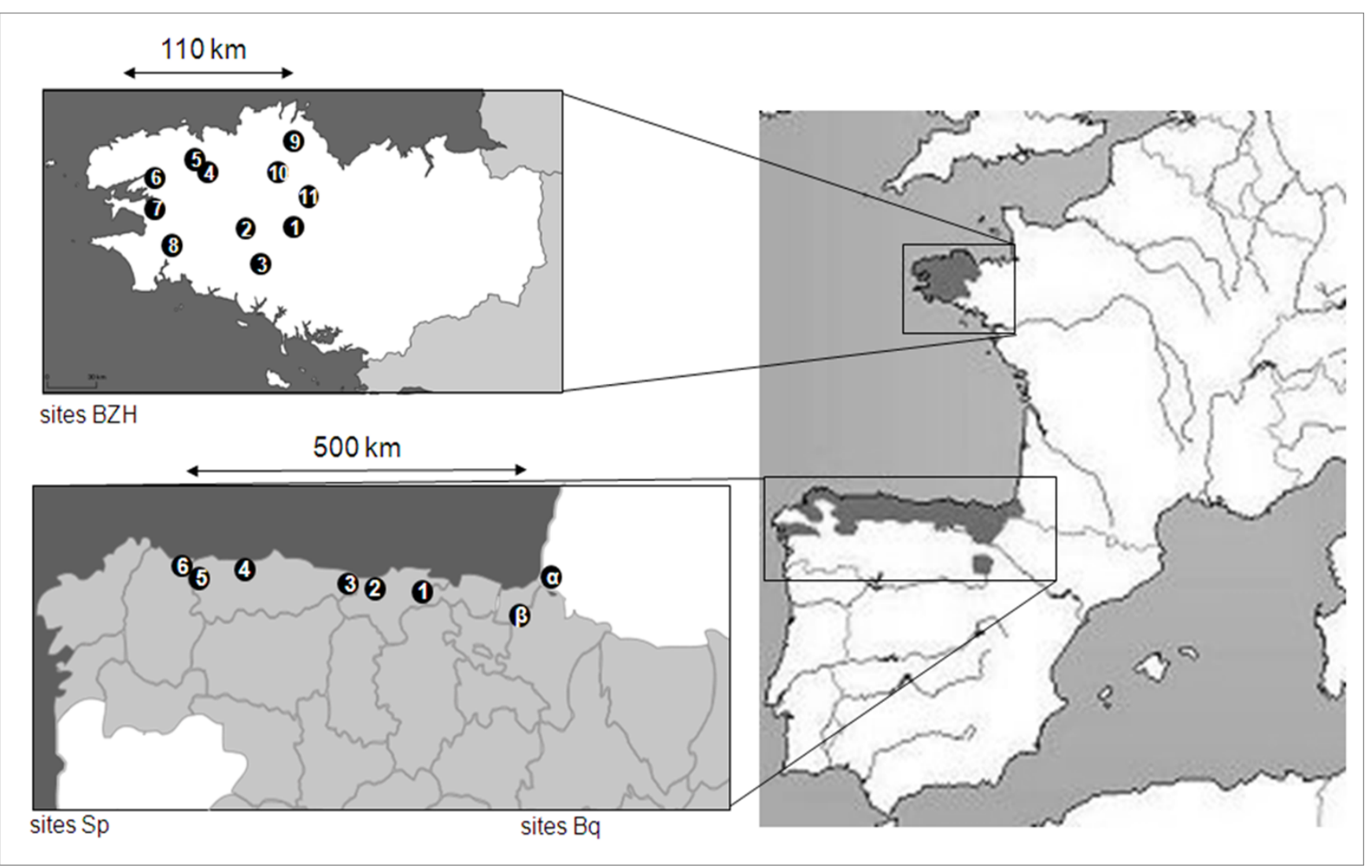

\section{Figure I}

Localities of the different populations sampled. In (i) Brittany (France), numbered from I to II, (ii) Spain, named from Spl to Sp8, and (iii) the Basque Country (France and Spain), called $\alpha$ and $\beta$. The samples cover the entire E. quimperiana distribution [25]. Modified from [75].

seven private haplotypes were found in five out of six populations: haplotypes 4 and 5 were typical of Sp6 (Reme), haplotype 6 was specific to Sp5 (Gio), haplotype 7 was only found at Sp4 (Pravia), haplotype 8 was present in Sp2 (Cobreces), and haplotypes 9 and 10 were typical for the Cantabria province (site Sp1, Ramales de la Victoria).

The second haplogroup, Hg11, comprised only one haplotype (H11), which only occurred in Basque snails from Bq $\alpha$ (Sare) and Bq $\beta$ (Toloza Betulu).

The populations of the two haplogroups formed by COI sequences ( $\mathrm{HgA}$ and $\mathrm{HgS}$ ) are the same as those found in Hg1 and Hg11 (Fig 4). HgA comprised 16 haplotypes (71 individuals), the most frequent of which was HA (44 individuals). The distribution of HA is comparable to that of $\mathrm{H} 1$, since it is found in both Brittany (except for the eastern sites, BZH1 and BZH11) and Spain (sites Sp3, Sp4 and Sp5). All of the other HgA haplotypes are represented by either a single or few specimens (Fig 5). The $\mathrm{HB}, \mathrm{HC}, \mathrm{HJ}$, HL and HO haplotypes are specific to Brittany. Most of the 10 haplotypes specific to Spain are private. As for haplo- group Hg11, HgW exclusively comprised all of the Basque snails and included seven private haplotypes (HQ, HR, HS, HT, HU, HV and HW).

The genetic diversity estimates are given in Table 1 . Obviously, both the haplotype and nucleotide diversities are much higher for both genes in the populations from Spain $(\mathrm{h} 16 \mathrm{~S}$ rDNA $=0.81 \pm 0.01 ; \pi 16 \mathrm{~S}$ rDNA $=1.89 \pm 1.25$; $\left.{ }^{\mathrm{h} C O I}=0.85 \pm 0.05 ; \pi \mathrm{COI}=4.73 \pm 2.39\right)$, compared to those from Brittany $(\mathrm{h} 16 \mathrm{~S}$ rDNA $=0.16 \pm 0.1, \pi 16 \mathrm{~S}$ rDNA $\left.=0.24 \pm 0.32 ;{ }^{\mathrm{h}} \mathrm{COI}=0.34 \pm 0.09 ;{ }^{\pi} \mathrm{COI}=0.44 \pm 0.4\right)$. While no variation is detected in the Basque populations for the $16 \mathrm{~S}$ region, the gene and nucleotide diversities are relatively high for the $\mathrm{COI}$ gene $(\mathrm{h} C \mathrm{COI}=0.91 \pm 0.07 ; \pi \mathrm{COI}$ $=3.89 \pm 2.11$ ).

\section{Demographic analysis}

Hypotheses of demographic expansion were tested using Fu's Fs and Ramos-Onsins \& Rozas' $R^{2}$ statistics (Table 2). Both tests yielded results that were consistent with a population expansion of both the Hg1 and HgA haplogroups, mainly driven by the significant expansion of the Brittany 


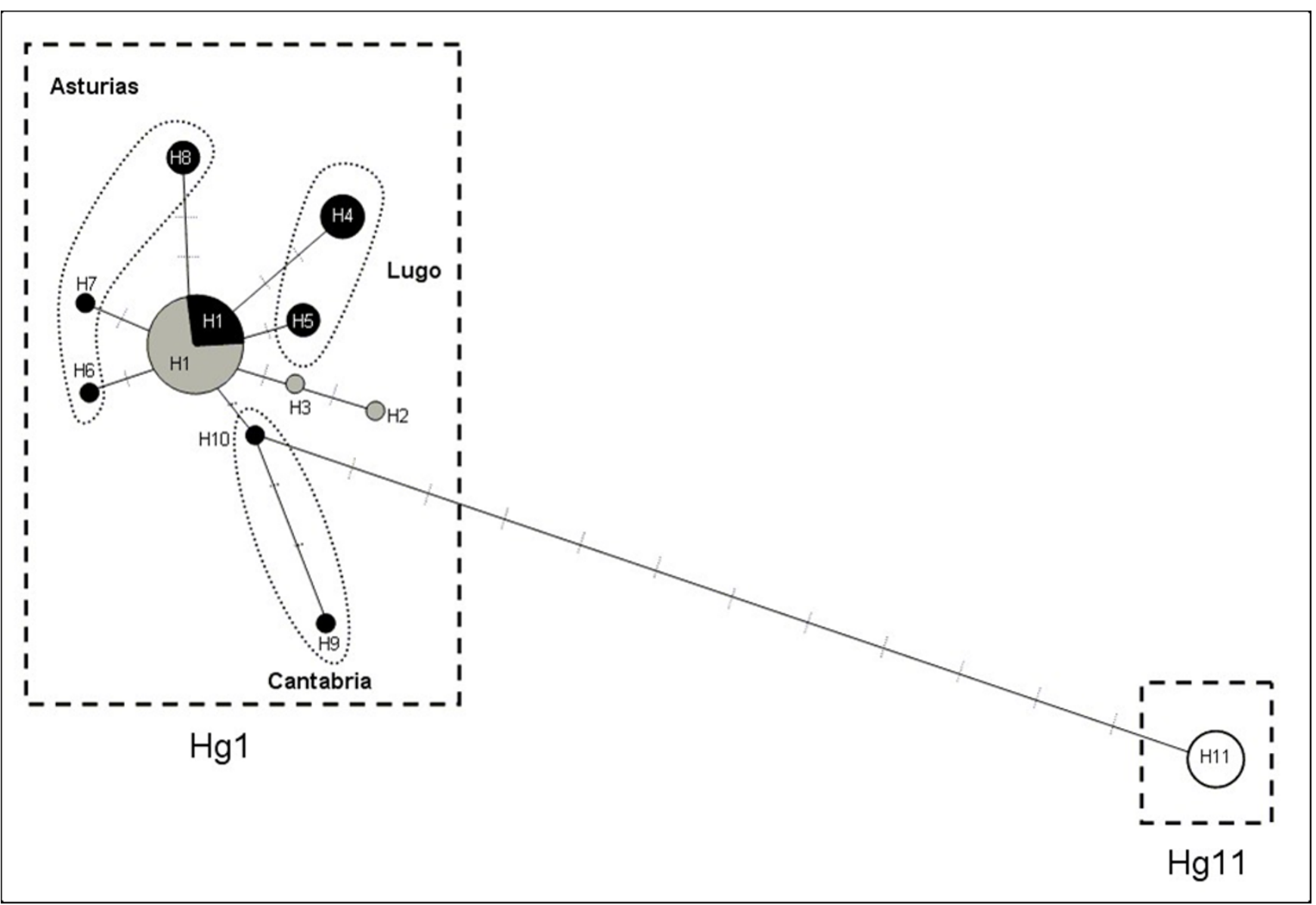

Figure 2

Unrooted haplotype networks of $\mathbf{E}$. quimperiana based on I 6S rDNA. Haplotypes originating from Brittany are represented in grey, those from Spain in black and those from the Basque Country in white. HI to HI I: names of the haplotypes; $\mathrm{HgI}$ and $\mathrm{Hgl} \mathrm{I}$ : names of the haplogroups. Haplotype $\mathrm{HI}$ is present in Spain in the Asturias province, while the province origin of the other haplotypes are specified on the figure (i.e. Asturias, Cantabria and Lugo).

populations. The unimodal distribution of pairwise differences among the Brittany populations (Fig 6) and the non-significant raggedness index obtained from the mismatch distribution analyses (Table 2 ) are consistent with a model of recent expansion [31].

\section{Bayesian estimation of time to the most recent common ancestor}

The molecular-clock likelihood ratio-tests (LRT) showed no significant variation of the substitution rate among the branches of both the COI and $16 \mathrm{~S}$ phylogenies. For $16 \mathrm{~S}$, $2 \Delta \operatorname{logL}=24.5, \mathrm{df}=54, \mathrm{p}=0.99 ;$ for COI, $2 \Delta \operatorname{logL}=29.5$, $\mathrm{df}=82, \mathrm{p}=0.99$ with all codon positions, and $2 \Delta \log \mathrm{L}=$ 66.33 , d.f. $=82, \mathrm{p}=0.90$, with the third-codon position only. Applying divergence rates ranging from 4 to $6.86 \%$ per Myr for the third codon of COI, the most recent common ancestor of all of the E. quimperiana populations (and 95\% HPD) would have lived between 1 (0.64-1.45) to 0.6 (0.37-0.84) Myr ago (Table 3). The TMRCA, based on the COI-3rd base divergence rate, was estimated as being between 0.43 and $0.25 \mathrm{Myr}$ for the Brittany-Spain lineage and between 0.31 and $0.18 \mathrm{Myr}$ for the Basque lineage. Convergent time estimates were obtained for the Brittany-Spain clade with a $2.2 \%$ rate of evolution for COI (all bases; $0.29 \mathrm{Myr}$ ) and 2\% for $16 \mathrm{~S}$ (0.20 Myr). For the Basque clade, while a divergence rate of $2.2 \%$ for COI (all bases) leads to the same time value estimate ( $0.18 \mathrm{Myr})$, a four times lower divergence rate for $16 \mathrm{~S}$ (around $0.5 \%$ / Myr) yielded an equivalent time estimate. In spite of homogeneous substitution rates among the branches in the $16 \mathrm{~S}$ phylogeny, the molecular clock does not seem to tick regularly.

\section{Discussion}

In spite of its name, an ancient Iberian origin of Elona quimperiana is obvious, as has been suggested earlier [26]. 


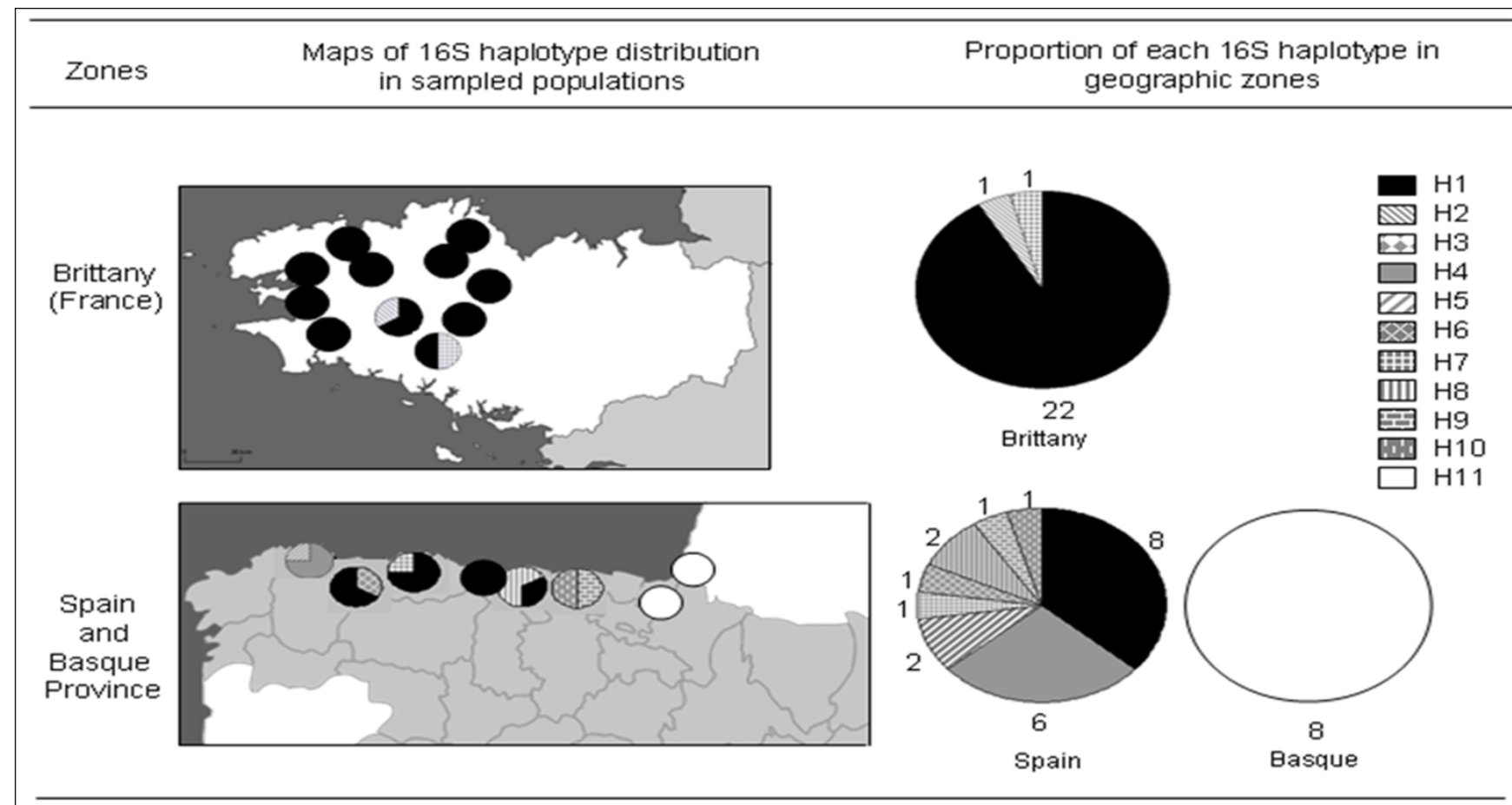

Figure 3

Geographic distribution of $E$. quimperiana haplotypes in sampled populations across Brittany, Spain and the Basque Country for I6S rDNA. Proportion of each haplotype in the different geographic zones is specified as the total number of individuals carrying these haplotypes.

This is strongly supported by the observation that most of the mtDNA diversity is found in Spain, and by the fact that Spanish haplotypes have a central position in both of the star-like sequence networks. Our genetic comparison of populations from the two disjunct distribution areas of E. quimperiana reveals that the genetic differentiation does not mirror the large geographical discontinuity of the species range.

Actually, the genetic divergences isolate the Basque populations from a homogeneous genetic group comprising the Spanish and Brittany populations. Both the 16S rDNA and COI genes show specific Basque haplotypes, forming separate clades in the two haplotype networks. The low level of genetic diversity, especially for the 16S gene, the occurrence of private haplotypes, and the peripheral location suggest that the Basque populations may form a parapatric race [14], although no morphological peculiarities could be detected. Such peripheral populations frequently mark a threshold of environmental variation, beyond which the species cannot expand [32]. As the split between the Basque and central Spanish populations would have occurred between 1 Myr to 600,000 BP, it can be most easily interpreted by assuming that the species survived the Pleistocene Ice Ages in separate glacial refugia on the Iberian Peninsula [16]. Indeed, the occurrence of 'refugia within an Iberian refugium' is now largely supported by data from a range of organisms. Among the seven putative terrestrial refugia identified in this area, those located along the Picos de Europa (north of Spain, [33-37]) and in/or near the Pyrenees [34,36,38] coincide quite strikingly with the phylogeographic structure we obtained (Fig 7). Our results thus support the spatial subdivision hypothesis of an Iberian Peninsula glacial refugia [16], and help to refine the Pyrenees refugium theory by suggesting the Basque Country as a refuge zone during the Pleistocene glaciations. Moreover, the presence of the closely related species Norelona pyrenaica, which is endemic in the eastern Pyrenees, where E. quimperiana does not live (Fig 7, [39]), suggests the occurrence of another separate refuge in this part of the Pyrenees, which is probably influenced by Mediterranean climates. Contemporary anthropogenic effects may also influence the population structure of E. quimperiana since estimates of the split of the Basque populations match glacial and postglacial times (Table 3). Recurrent bottlenecks, due to habitat fragmentation and the alteration of natural forest habitat in the Basque Country [40], could have accentu- 


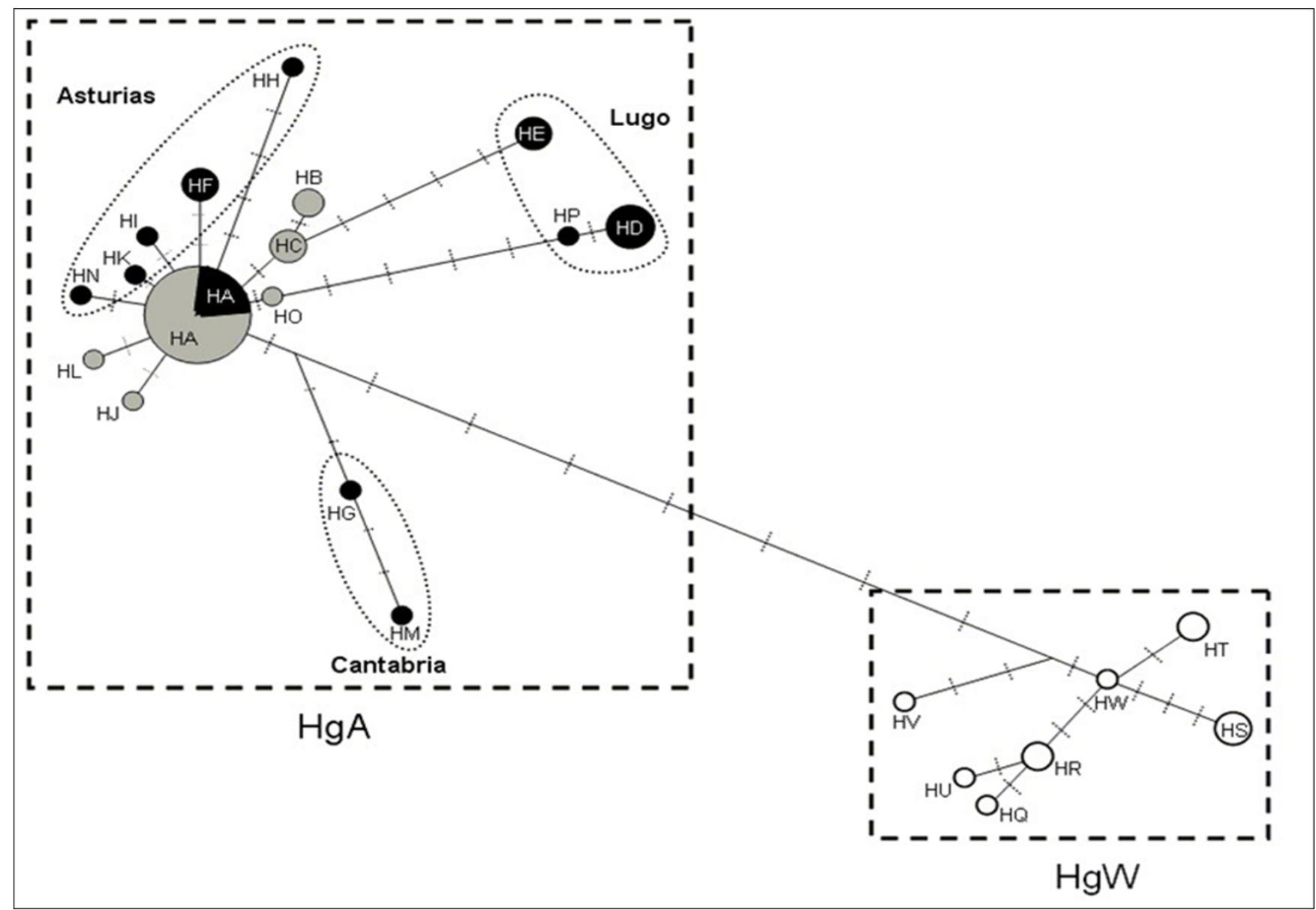

\section{Figure 4}

Unrooted haplotype networks of E. quimperiana based on COI genes. Haplotypes originating from Brittany are represented in grey, those from Spain in black and those from the Basque Country in white. HA to HW: names of the haplotypes; $\mathrm{HgA}$ and $\mathrm{HgW}$ : names of the haplogroups. Haplotype $\mathrm{HA}$ is present in Spain in the Asturias province, while the province origin of the other haplotypes are specified on the figure (i.e. Asturias, Cantabria and Lugo).

ated this genetic divergence (e.g. $[41,42])$. This could explain the discrepancies in the divergence time estimates between the two genetic markers, while a strong concordance has been observed in the divergence time estimates for other E. quimperiana lineages. By harboring distinct haplotypes, the Basque Country populations appear to be of great importance in terms of potential adaptation, long term persistence and hence, the conservation of E. quimperiana.

Besides the strong genetic divergence of the Basque lineage, the present results also show that in the Spain-Brittany lineage, (i) the genetic diversity is higher in Spain, (ii) the Spanish and Brittany populations share one common haplotype for each gene, which is, moreover, the most frequent in the two areas, and (iii) the Spanish populations are at demographic equilibrium, while the Brittany ones would have experienced a recent expansion.
Three scenarios might be considered in order to explain the origin of Brittany populations: 1 - a recent introduction by human activities, 2 - an ancient northward colonization preceding the last glacial period, as demonstrated for other Lusitanian species [19], 3 - a spatial expansion after the Last Glacial Maximum (LGM), also from native Spanish populations [24]. In the first case, it is expected to have haplotypes in common with the source and introduced zones, as illustrated by $16 \mathrm{~S}$ rDNA-H1 and COI-HA. All of the other haplotypes found in Brittany are specific to this area, suggesting that they probably appeared in Brittany after an introduction. However, such post-introduction genetic diversification is more typical for species with high colonization capabilities, which is not the case for E. quimperiana [29]. Moreover, related life history features, inconsistent with human activities or production, render multiple introductions improbable. All of these points make the first assumption rather unlikely. 


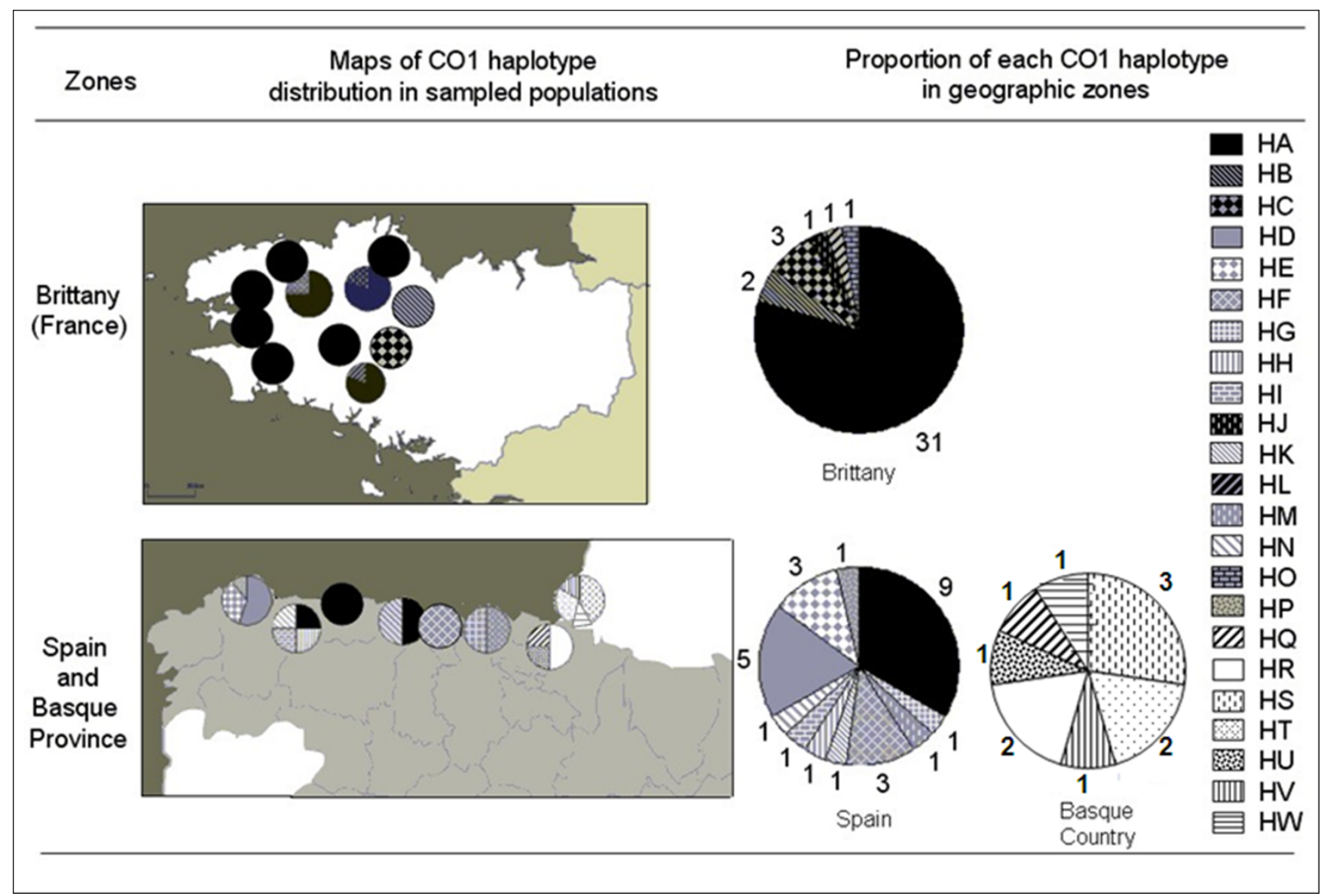

Figure 5

Geographic distribution of $E$. quimperiana haplotypes in sampled populations across Brittany, Spain and the Basque Country for COl genes. Proportion of each haplotype in the different geographic zones is specified as the total number of individuals carrying these haplotypes.

The second hypothesis conforms to predictions of the effects of the ice ages on genetic variability in populations: populations with historical distributions in northern latitudes present lower genetic diversity, with fewer differentiated lineages, than southern populations (Hewitt [14] and Avise [9]). This has been demonstrated in other ecto- therm species [43]. An ancient expansion of populations from Iberia through France during the Pleistocene interglacial periods may be plausible, since during these periods, the environmental conditions in Western Europe (e.g. high precipitation and cool temperatures) were particularly favorable to E. quimperiana, all the more so given

Table I: Genetic polymorphism for different subsets of $E$. quimperiana populations according to their geographic origin.

\begin{tabular}{|c|c|c|c|c|c|c|c|c|}
\hline \multirow[t]{2}{*}{ Population } & \multicolumn{2}{|c|}{$N_{\text {haplotype }}$} & \multicolumn{2}{|c|}{$N_{\text {ind }}$} & \multicolumn{2}{|c|}{$h(S D)$} & \multicolumn{2}{|c|}{$\pi(S D)$} \\
\hline & 165 & $\mathrm{COI}$ & 165 & $\mathrm{COI}$ & 165 & $\mathrm{COI}$ & $16 S$ & $\mathrm{COI}$ \\
\hline \multirow[t]{2}{*}{ Brittany } & 3 & 6 & 24 & 43 & 0.16 & 0.34 & 0.24 & 0.44 \\
\hline & & & & & $(0.10)$ & $(0.09)$ & $(0.32)$ & $(0.40)$ \\
\hline \multirow[t]{2}{*}{ Spain } & 8 & 11 & 22 & 27 & 0.81 & 0.85 & 1.89 & 4.73 \\
\hline & & & & & $(0.01)$ & $(0.05)$ & $(1.25)$ & (2.39) \\
\hline \multirow[t]{2}{*}{ Basque Country } & 1 & 7 & 8 & 11 & 0.00 & 0.91 & 0.00 & 3.89 \\
\hline & & & & & $(0.00)$ & $(0.07)$ & $(0.00)$ & $(2.11)$ \\
\hline \multirow[t]{2}{*}{ All } & 11 & 23 & 54 & 81 & 0.67 & 0.70 & 3.685 .57 & \\
\hline & & & & & $(0.07)$ & $(0.06)$ & $(2.2 I)$ & (2.70) \\
\hline
\end{tabular}

$\mathrm{N}_{\text {haplotypes: }}$ number of haplotypes; $\mathrm{N}_{\text {ind }}$ : number of individuals; h: haplotype diversity; $\pi$ : nucleotide diversity; SD: standard deviation. 
Table 2: Demographic analyses testing hypothesis of $E$. quimperiana population expansion.

\begin{tabular}{|c|c|c|c|c|c|c|c|c|c|c|}
\hline \multirow[t]{2}{*}{ Statistics } & \multicolumn{2}{|c|}{ All } & \multicolumn{2}{|c|}{ Brittany } & \multicolumn{2}{|c|}{ Spain } & \multicolumn{2}{|c|}{ Brittany-Spain } & \multicolumn{2}{|c|}{ Basque } \\
\hline & $16 S$ & $\mathrm{COI}$ & 165 & $\mathrm{COI}$ & 165 & $\mathrm{COI}$ & $16 S$ & $\mathrm{COI}$ & $16 S$ & $\mathrm{COI}$ \\
\hline Fu's FS & -3.89 & $-|.4|$ & -3.90 & -2.16 & -0.8 & -5.04 & -5.96 & 0.00 & -0.98 & \\
\hline FS p-value & 0.56 & 0.13 & $0.03 *$ & $0.003 * * *$ & 0.11 & 0.39 & $0.002 * * *$ & $0.01 * *$ & N.A. & 0.24 \\
\hline Ramos-Onsins \& ozas'R² & 0.06 & 0.11 & 0.06 & 0.09 & 0.09 & 0.05 & 0.04 & N.A. & 0.15 & \\
\hline $\mathrm{R}^{2} p$-value & 0.32 & 0.10 & 0.14 & $0.05^{*}$ & 0.09 & 0.15 & $0.02 *$ & $0.02 *$ & N.A. & 0.28 \\
\hline Raggedness index $r$ & 0.11 & 0.05 & 0.57 & 0.21 & 0.05 & 0.05 & 0.12 & 0.08 & N.A. & 0.15 \\
\hline$r$-value & 1 & 0.76 & 0.73 & 0.65 & 0.49 & 0.48 & I & 1 & N.A. & 0.14 \\
\hline
\end{tabular}

Analyses are using Fu's Fs and Ramos-Onsins \& Rozas' $R^{2}$ statistics, for the whole sample (all) and four subsets of E. quimperiana populations (no sign: non-significant; *: p-value < 0.05; ***: p-value $<0.01$ ).
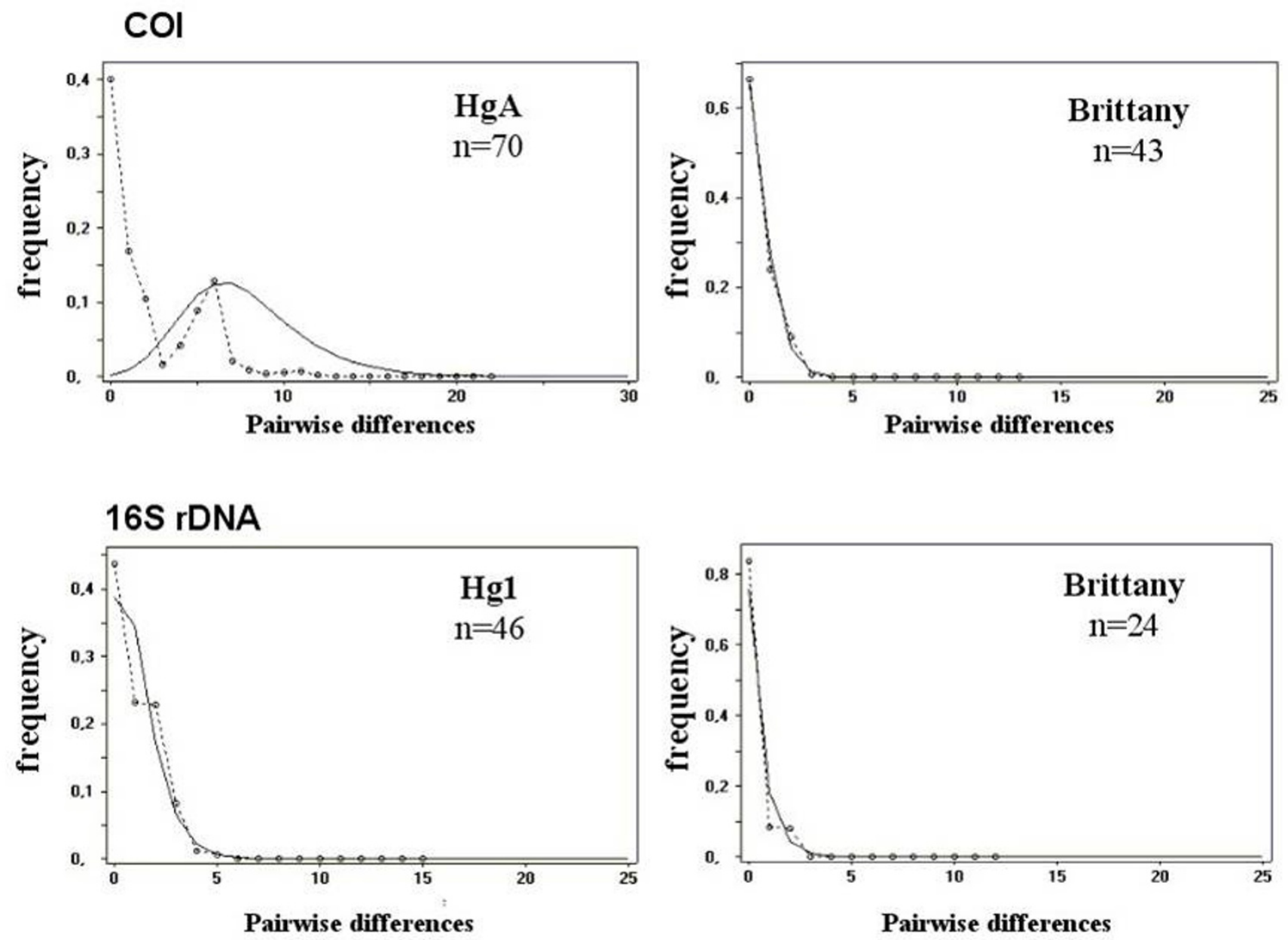

\section{Figure 6}

Mismatch distribution of substitution differences between pairs of individuals from Brittany for I6S rDNA and COI genes. The observed frequency is given as a dotted line. The expected distribution under a growth-decline model determined using the DNASP v3.5 program [54] is represented by a continuous line. 
Table 3: Time to the most recent common ancestor (TMRCA; in million years, Myr) for all E. quimperiana sequences and for the two phylogenetic lineages defined.

\begin{tabular}{|c|c|c|c|c|c|}
\hline \multirow[t]{2}{*}{ Gene } & \multirow{2}{*}{\multicolumn{2}{|c|}{ Standard molecular clock estimates }} & \multicolumn{3}{|c|}{ Divergence time estimates } \\
\hline & & & All & Brittany-Spain & Basque Country \\
\hline \multirow[t]{15}{*}{ COI } & 4\% per Myr & mean (in Myr) & 1.02 & 0.43 & 0.31 \\
\hline & (third base) & $95 \%$ hpd upper & 0.64 & 0.25 & 0.14 \\
\hline & & $95 \%$ hpd lower & 1.45 & 0.65 & 0.52 \\
\hline & $6.86 \%$ per Myr & mean (in Myr) & 0.60 & 0.25 & 0.18 \\
\hline & (third base) & $95 \%$ hpd upper & 0.37 & 0.14 & 0.08 \\
\hline & & $95 \%$ hpd lower & 0.84 & 0.38 & 0.30 \\
\hline & 2\% per Myr & mean (in Myr) & 0.65 & 0.31 & 0.20 \\
\hline & (three bases) & $95 \%$ hpd upper & 0.42 & 0.17 & 0.09 \\
\hline & & $95 \%$ hpd lower & 0.92 & 0.46 & 0.34 \\
\hline & $2.2 \%$ per $M y r$ & mean (in Myr) & 0.59 & 0.29 & 0.18 \\
\hline & (three bases) & $95 \%$ hpd upper & 0.38 & 0.15 & 0.08 \\
\hline & & $95 \%$ hpd lower & 0.84 & 0.42 & 0.31 \\
\hline & 3\% per Myr & mean (in Myr) & 0.44 & 0.21 & 0.13 \\
\hline & (three bases) & $95 \%$ hpd upper & 0.28 & 0.11 & 0.06 \\
\hline & & $95 \%$ hpd lower & 0.61 & 0.31 & 0.23 \\
\hline \multirow[t]{9}{*}{$16 S$} & $0.5 \%$ per $\mathbf{M y r}$ & mean (in Myr) & 2.39 & 0.81 & 0.17 \\
\hline & & $95 \%$ hpd upper & 1.30 & 0.30 & 0.01 \\
\hline & & $95 \%$ hpd lower & 3.70 & 1.36 & 0.41 \\
\hline & $2 \%$ per $M y r$ & mean (in Myr) & 0.60 & 0.20 & 0.04 \\
\hline & & $95 \%$ hpd upper & 0.32 & 0.08 & 0.002 \\
\hline & & $95 \%$ hpd lower & 0.95 & 0.34 & 0.10 \\
\hline & $10 \%$ per Myr & mean (in Myr) & 0.12 & 0.04 & 0.01 \\
\hline & & $95 \%$ hpd upper & 0.06 & 0.02 & 0.0004 \\
\hline & & $95 \%$ hpd lower & 0.19 & 0.07 & 0.02 \\
\hline
\end{tabular}

Results of Bayesian analyses are based on I6S rRNA and COI (considering the three bases or the third-codon position only) variation.

that oak was a dominant tree species [30]. Under this hypothesis, a subset of southern haplotypes (from the Picos de Europa refugium) would have expanded northward, while others (from the Pyrenees refugium) remained in the south. Glaciations would have subsequently eradicated most of the E. quimperiana populations, except in the Iberian refugia and in a northern refuge zone. Indeed, small microenvironmentally favorable zones might have been created by oak trees in Western Europe [44]. According to paleoclimatic reconstructions, the local environmental conditions in close proximity to the arboreal vegetation were indeed warmer and more humid than regional simulations of the full-glacial climate [45]. Within close proximity to the trees, poor coldhardy flora and fauna were also able to survive the extremes of the LGM. It is also possible that the Pleistocene refuge in northwestern France may involve caves, as suspected in Belgium for other poor cold-hardy animals and deciduous trees [18]. Elona quimperiana is indeed frequently observed in caves [46], where it survives by coprophagous and necrophagous feeding.

Unfortunately, our results cannot distinguish between the second and the third scenario. The latter implies a colonization of Brittany after the last glaciations. As illustrated by oak (Quercus sp.) dispersal in Great Britain some 10
000-9500 years ago [47], the oceanic climate of the west coast in France could have allowed a rapid spread of $E$. quimperiana from southern refugia to northern areas. Since this snail is strongly associated with oak forests, its dispersal may follow the tree expansion pattern. Human deforestation in intermediate areas between the Basque Country and Brittany, around 1000-500 years ago, may have created induced open habitats that are hostile for $E$. quimperiana [48], thus potentially explaining its present disjunct distribution.

There is no fossil record of E. Quimperiana due to the extreme fragility of its thin shell. Although the occurrence of a northern refugium should not be discarded a priori, an exhaustive sampling of the southern distribution range (based on a new imperative inventory, since many recorded sites appear to be presently disturbed by human activities) where Pleistocene refugia are obvious, appears necessary in order to clarify the colonization history of Brittany by E. quimperiana.

\section{Conclusion}

The present results confirm the Iberian origin of the land snail E. quimperiana and reveal a genetic differentiation separating a Basque lineage from monophyletic clade grouping sequences from Brittany and Spain. Divergence 


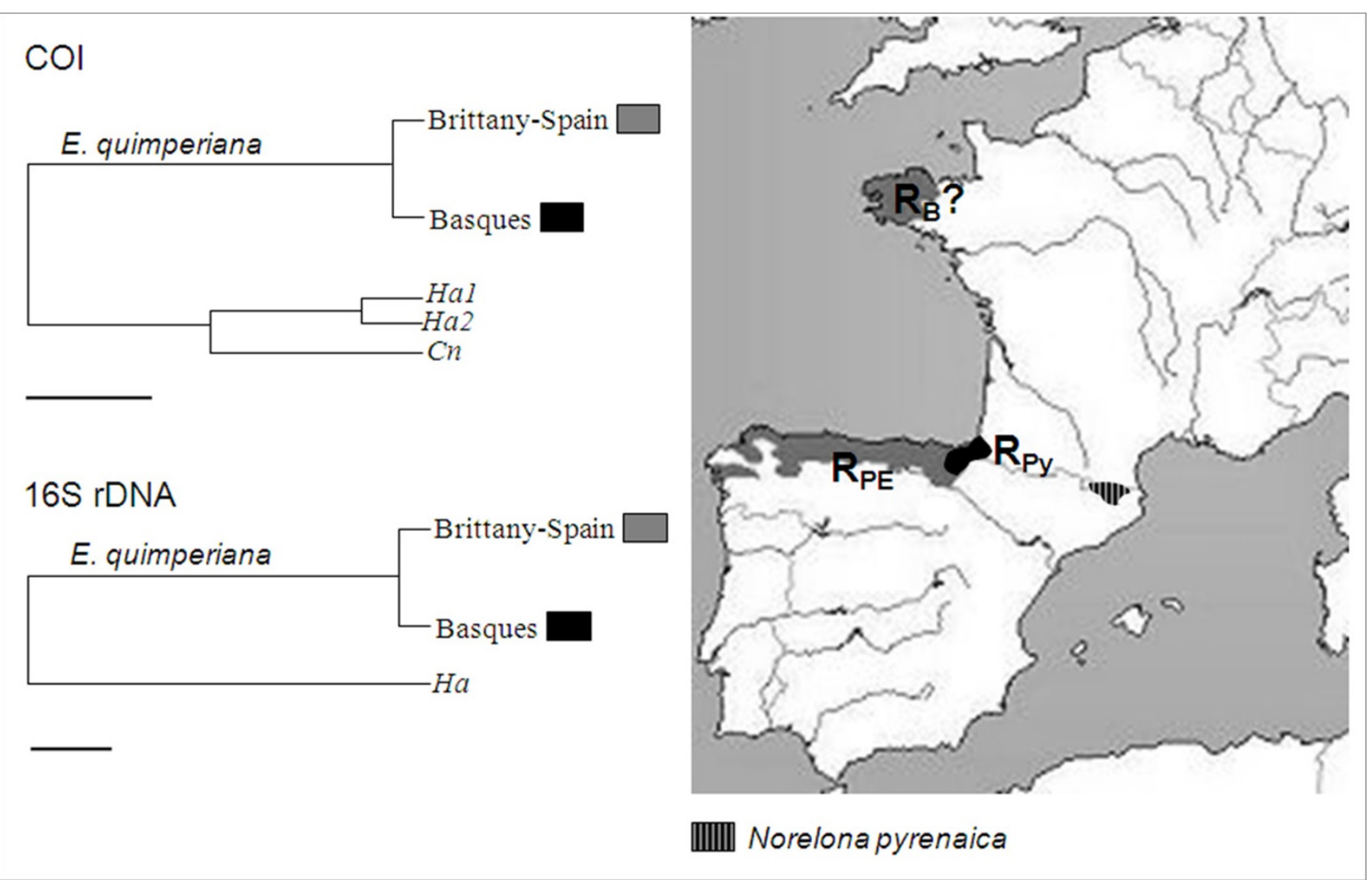

Figure 7

Synthetic representation of the phylogenetic structure of E. quimperiana populations from Brittany, Spain and the Basque Country. $\mathrm{Ha}$ and $\mathrm{Cn}$ : outgroups corresponding respectively to Cantareus aspersus and Cepaea nemoralis. The scaled bar under the trees represents a $0.1 \%$ sequence divergence. Genetically sustained refugia during the glaciations are localized in Picos de Europa in Spain $\left(R_{P E}\right)$ and the Basque Country in the Pyrenees $\left(R_{P Y}\right)$, while a putative third refugia during the last glaciations may be hypothesized in Brittany $\left(R_{B}\right.$ ?). The localized distribution of the related species Norelona pyrenaica is specified (eastern Pyrenees).

time estimates suggest the coexistence of these lineages in the Iberian refugia during the LGM, and strongly support the hypothesis of 'refugia in refugia' during glaciations. The identification of Iberian lineages allows the precision of the glacial refugia in the Basque Country and Asturias locations. Moreover, the species' ecology (inhabiting deciduous oak forests) allows researchers to sharpen their focus on the type of habitat present in these refugia during the glaciations. The scenario of a spatial expansion of $E$. quimperiana from the Iberian refugia located in Asturias to northern areas in France is the most probable for explaining the present distribution of the land snail. Inferences based on a spatial genetic structure analysis should be helpful to discriminate between a pre-glacial expansion implying the existence of glacial refugia in Brittany, or a post-glacial expansion followed by the human oak deforestation along the Atlantic coast in France.

\section{Methods \\ Sample collection}

Tissue samples were taken from a total of 81 individuals legally collected in 19 field populations across Brittany (France, sites BZH1 to BZH11), the Basque Country (France and Spain, sites $\mathrm{BQ} \alpha$ and $\mathrm{BQ} \beta$ ) and Northern Spain (sites Sp1 to Sp6) between 2006 and 2007 (Fig 1). The other sites where this species was previously inventoried (see [26] for a review) were thoroughly searched, but no snails were found. The principal threat to this species is probably deforestation in those areas.

\section{DNA Extraction, Amplification and Sequencing}

Foot tissue was dissected from each individual and placed in a $10 \%$ chelex solution, with $15 \mu \mathrm{l}$ of proteinase $\mathrm{K}(10$ $\mathrm{mg} / \mathrm{ml}$ ). Samples were incubated overnight at $55^{\circ} \mathrm{C}$, and then briefly vortexed, before being boiled for $2 \times 15 \mathrm{~min}$. 
After a centrifugation at $10,000 \mathrm{~g}$ for $5 \mathrm{~s}$, the DNA in the supernatant was used in the subsequent amplifications.

DNA was amplified by the polymerase chain reaction (PCR) for the mitochondrial 16S rDNA on a set of 54 individuals, and mitochondrial cytochrome $c$ oxydase-1 (COI) on 81 individuals. The 16S rDNA and COI gene fragments correspond, respectively, to a 480 base pair sequence using the primers 984 (5'-CGCCTGTTTAACAAAAACAT-3') and 16S2 (5'-CTGGCTTACGCCGGTCTG-3') [49], and to a 683 base pair sequence using the primers FCOI (5'-ACTCAACGAATCATAAAGATATTGG$\left.3^{\prime}\right)$ and RCOI (5'-TATACTTCAGGATGACCAAAAAATCA$\left.3^{\prime}\right)$ [50,51]. PCRs were carried out in $25 \mu$ l volumes containing $0.5 \mu \mathrm{l}(10 \mu \mathrm{M})$ of each forward and reverse primers, $12.5 \mu \mathrm{l}$ of the Diamond DNA Polymerase - 500 $\left(\right.$ Bioline $\left.^{\varpi}\right), 10.25 \mu \mathrm{l}$ of UP water and $1.25 \mu \mathrm{l}$ of DNA template. The PCR conditions for the 16S rDNA gene were an initial denaturation step of $94^{\circ} \mathrm{C}(3 \mathrm{~min})$, followed by 35 cycles of $94^{\circ} \mathrm{C}(30 \mathrm{~s}), 49^{\circ} \mathrm{C}(30 \mathrm{~s}), 72^{\circ} \mathrm{C}(40 \mathrm{~s})$ and a final extension phase at $72^{\circ} \mathrm{C}$ for $4 \mathrm{~min}$. Those for the COI gene were an initial denaturation step of $94^{\circ} \mathrm{C}(5 \mathrm{~min})$, followed 35 cycles of $94^{\circ} \mathrm{C}(45 \mathrm{~s}), 52^{\circ} \mathrm{C}(45 \mathrm{~s}), 72^{\circ} \mathrm{C}(1$ $\min$ ) and a final extension phase at $72^{\circ} \mathrm{C}$ for $7 \mathrm{~min}$. The PCR products were sequenced in both directions on an automated sequencer using the PCR primers (PE Applied Biosystems 310 Genetic Analyser, UMR 6553; plate-forme de séquençage génotypage OUEST-genopole ${ }^{\circledast}$ ).

\section{Sequence analysis}

Sequences were aligned using CLUSTAL W [52] and were manually checked in the BIOEDIT sequence editing program [53]. The sequences have been submitted to GenBank (Accession N $N^{\circ}$ FJ491809-FJ491943). Sequence polymorphism analyses (haplotype diversity $h$ and nucleotide diversity $\pi$ ) were carried out with DNASP version 4.10. [54] and ARLEQUIN version 3.1 [55]. The land snails Cantareus aspersus and Cepaea nemoralis were used as outgroups.

\section{Phylogenetic analysis}

Phylogenetic relationships among haplotypes were estimated using maximum likelihood (ML) and MarkovChain Monte-Carlo (MCMC) bayesian-based inference (BI) methods. ML and BI analyses were performed using PAUP* version 4.0b10 [56] and MRBAYES v. 3.1.1-p1 [57] softwares, respectively. The best fit model of nucleotide substitutions was selected prior to ML and BI using the Akaike Information Criterion [58]. The software MrAIC v.1.4.2 [59] was used to evaluate the fit of the data to 24 different models of nucleotide substitutions. The resulting best fit model was the F81 model [60] for the 16S rDNA region, with an unequal rate for base frequencies and equal rates for transitions and transversion. For the COI gene, the best fit model was the HKY model [61] with two different rates for transitions and transversion, unequal base frequencies, a parameter for invariable sites $(I)$ and a gamma distribution parameter that describes the rate variation across variable sites $(I)$. The model of nucleotide substitution was incorporated in PHYML[62] and in MRBAYES for the ML and BI analyses respectively. For the ML analysis, the robustness of inferences was assessed by bootstrap resampling using 1,000 repetitions. For the Bayesian analysis, the posterior probabilities of trees and parameters were approximated with Markov-Chain Monte-Carlo and Metropolis coupling. We ran two independent MCMC analyses with four chains and a temperature set to 0.2. Each chain was run for 2,000,000 cycles with trees sampled every 100 generations. Posterior probabilities were obtained from the $50 \%$ majority rule consensus of trees sampled after discarding the trees saved before chains reached apparent stationarity (i.e. a 'burn-in period" of 50,000 generations for 16S rDNA).

We used network-based approaches for intraspecific phylogenetic analyses to deal with the low divergence among individuals and the possible persistence of ancestral nodes [63]. The median joining algorithm implemented in the NETWORK v. 4.2.0.1 software [64] was used with the default settings for constructing networks (weight $=10$ and $\mathbf{e}=0$ ), and we simplified the median networks that contained all of the possible equally shortest trees by running the MP (maximum parsimony) calculation option.

\section{Demographic analyses}

The demographic history of the populations was inferred using two of the most powerful tests of population expansion: (i) Fu's Fs [65], which represents the probability of observing a similar or a higher number of haplotypes in a random neutral population given the observed value of theta. In populations that have experienced recent expansion, large negative values of $F s$ are expected, due to an excess of rare alleles; (ii) Ramos-Onsins \& Rozas' [66] $R^{2}$ statistics, representing the difference between the number of singleton mutations and the average number of pairwise differences. Recent population expansions are expected to be associated with low values of this parameter. $R^{2}$ and $F s$ statistics were estimated using DNASP v. 4.10.9 [54] and ARLEQUIN v. 3.1 [55] respectively, and their significance was assessed using 1,000 coalescent simulated resamplings. In order to evaluate the possible historical events of population growth or decline, mismatch distribution analyses were also performed [67]; in the first case, populations show unimodal distributions, while in the second case the populations at demographic equilibrium present multimodal distributions [68]. Mismatch distributions were computed for each haplogroup and compared to the expected distributions obtained under a model of sudden expansion. 


\section{Molecular clock and divergence times}

A molecular-clock likelihood ratio-test (LRT, $[47,50])$ was first conducted on the $16 \mathrm{~S}$ rDNA and COI sequences (considering the three bases and only the third base of codon) to ascertain whether or not the Elona sequences were evolving at a homogeneous rate along all of the branches in the phylogenies. This test compares the loglikelihood of the ML trees under alternative molecular clock assumptions (i.e. a relaxed vs. an enforced molecular clock). The statistic A = 2(lnL1-lnL2) could be compared with a distribution with $(n-2)$ degrees of freedom (where $n$ is the number of sequences). Divergence dates were computed using BEAST[69] in order to provide estimates of time to the most common ancestor (TMCRA) for E. quimperiana populations, along with the TMCRAs of the Brittany-Spain (Hg1 and HgA) and the Basque lineages (see Results). An HKY model of nucleotide substitution was employed with a sequence divergence rate based on several different published rates for molluscs. While the divergence rates for $16 \mathrm{~S}$ rDNA are estimated at around $2 \%$ per Myr for animals [70], the estimates for gastropods vary between $0.5-0.6 \%[71,72]$ up to an accelerated rate of $10 \%$ per Myr, as seen in the land snail Mandarina sp [73]. For COIGENE, we used rates from bivalve species inferred from geological events [74]. These rates range from 0.03 to $6.84 \%$ per Myr, depending on the codon position considered. The number of mutations recorded for the COI first- and second-positions (7 of 456 nucleotides) and COI third-positions (39 of 227 nucleotides) may reflect a variation in rates of substitution among these types of sites, in favor of a higher rate for COI third-positions. Given that the clades of the BI tree, based on the two first codon positions of COI, appeared to not be statistically supported (result not shown), we applied COI third-positions rates only when estimating divergence times ( 4 to $6.86 \%$ per Myr). These time estimates were then used to infer the $16 \mathrm{~S}$ rDNA mutation rates by testing several substitution rates $(0.5 \%, 2 \%$ and $10 \%$ per Myr).

\section{Authors' contributions}

$\mathrm{AV}$ and LM conceived and designed the research; AV, AB and LM conducted the samplings; AV performed the DNA extractions, AG and AV analyzed the data, and AV and AG wrote the paper.

\section{Acknowledgements}

We thank M.A. Coutellec and Ana Puente for their help with the sample collection, Olivier Lemoine and anonymous referees for their comments on the results.

\section{References}

I. Comes HP, Kadereit JW: The effect of Quaternary climatic changes on plant distribution and evolution. Trends Plant Sci 1998, 3:432-438.

2. Sokal RR, Thomson BA: Spatial genetic structure of human populations in Japan. Hum Biol 1998, 70:1-22.

3. Keyghobadi N, Roland J, Strobeck C: Genetic differentiation and gene flow among populations of the alpine butterfly, Parnas- sius smintheus, vary with landscape connectivity. Mol Ecol I999, 8:148I-1495.

4. Castric V, Bonney F, Bernatchez L: Landscape structure and hierarchical genetic diversity in the brook charr, Salvelinus fontinalis. Evolution 2001, 55:1016-1026.

5. Hirao AS, Kudo G: Landscape genetics of alpine-snowbed plants: comparisons along geographic and snowmelt gradients. Heredity 2004, 93:290-298.

6. Funk WC, Greene AE, Corn PS, Allendorf FW: High dispersal in a frog species suggests that it is vulnerable to habitat fragmentation. Biol Lett 2005, I:13-16.

7. Thomas JA, Morris MG: Patterns, mechanisms and rates of decline among UK invertebrates. Philos Trans R Soc Lond B 1994, 344:47-54.

8. Brookes MI, Graneau YA, King P, Rose OC, Thomas CD, Mallet JLB: Genetic analysis of founder bottlenecks in the rare British butterfly Plebejus argus. Conserv Biol 1997, I I:648-66I.

9. Avise JC, Walker D, Johns GC: Speciation durations and Pleistocene effects on vertebrate phylogeography. Proc $R$ Soc Lond B 1998, 265(1407): 1707-17|2.

10. Taberlet P, Fumagalli L, Wust-Saucy A, Cosson J: Comparative phylogeography and postglacial colonisation routes in Europe. Mol Ecol 1998, 7:453-464.

II. Hewitt GM: Post-glacial re-colonisation of European biota. Biol J Linn Soc 1999, 68:87-II 2.

12. Hewitt GM: The genetic legacy of the Quaternary ice ages. Nature 2000, 405:907-913.

13. Hewitt GM: Genetic consequences of climatic oscillations in the Quaternary. Philos Trans R Soc Lond B 2004, 359:183-195.

14. Hewitt GM: Some genetic consequences of ice ages, and their role in divergence and speciation. Biol J Linn Soc 1996, 58:247-276.

15. Michaux JR, Magnanou E, Paradis E, Nieberding C, Libois RM: Mitochondrial phylogeography of the woodmouse (Apodemus sylvaticus) in the western Palearctic region. Mol Ecol 2003, 1 2:685-697.

16. Gomez A, Lunt DH: Refugia within refugia: patterns of phylogeographic concordance in the Iberian Peninsula. Phylogeography of Southern European Refugia 2007:155-188. [Netherlands]

17. Martinkova N, McDonald RA, Searle JB: Stoats (Mustela erminea) provide evidence of natural overland colonisation of Ireland. Proc R Soc Lond B 2007, 274:1387-1393.

18. Stewart JR, Lister AM: Cryptic northern refugia and the origins of the modern biota. Trends Ecol Evol 200I, 16:608-6I3.

19. Rowe G, Harris DJ, Beebee TJC: Lusitania revisited: A phylogeographic analysis of the natterjack toad Bufo calamita across its entire biogeographical range. Mol Phylogenet Evol 2006, 39(2):335-346.

20. Renssen $\mathrm{H}$, Vandenberghe J: Investigation of the relationship between permafrost distribution in NW Europe and extensive winter sea-ice cover in the North Atlantic Ocean during the cold phases of the Last Glaciation. Quat Sci Rev 2003, 22:209-223.

21. Hanfling B, Hellemans B, Volckaert FAM, Carvalho R: Late glacial history of the cold-adapted freshwater Cottus gobio, revealed by microsatellites. Mol Ecol 2002, I I:1717-1729.

22. West RG: Plant Life of the Quaternary Cold Stages. Cambridge University Press; 2000.

23. Michaud JR, Hardy OJ, Justy F, Fournier P, Kranz A, Cabria M, Davison $A$, Rosoux R, Libois R: Conservation genetics and populations history of the threatened European mink Mustela lutreola, with emphasis on the west European population. Mol Ecol 2005, 14:2373-2388.

24. Preece RC, Coxon P, Robinson JE: New biostratigraphic evidence of the post-glacial colonisation of Ireland and for Mesolithic forest disturbance. J Biogeogr 1986, 13:487-509.

25. Gittenberger E: On Elona (Pulmonata, Elonidae Fam. Nov). Malacologia 1979, I 8: I39-145.

26. Puente Al, Altonaga K: Revision de las especies ibericas de la familia Xanthonychidae (Gastropoda Pulmonata: Helicoidea). Butll Inst Cat Hist Nat 1995, 63:85-101.

27. Caziot E: La faune terrestre lusitanienne. Ann Soc Linn Lyon 1915, 62:43-65.

28. Vincent P: The Biogeography of the British Isles. Routledge, London 1990. 
29. Daguzan J, Gloaquen JC: Contribution à l'écologie d'Elona quimperiana (de Férussac) (Gastéropode Pulmoné Stylommathophore) en Bretagne occidentale. Haliotis 1986, 15:17-30.

30. Cheddadi R, de Beaulieu JL, Jouzel J, Andrieu-Ponel V, Laurent J-M, Reille M, Raynaud D, Bar-Hen A: Similarity of vegetation dynamics during interglacial periods. Proc Natl Acad Sci USA 2005 102: I3939-13943.

31. Harpending HC: Signature of ancient population growth in a low-resolution mitochondrial DNA mismatch distribution. Hum Biol 1994, 66:591-600.

32. Böhme MU, Schneeweiss N, Fritz U, Schlegel M, Berendonk TU: Small edge populations at risk: genetic diversity of the green lizard (Lacerta viridis viridis) in Germany and implications for conservation management. Conserv Genet 2007, 8:555-563.

33. Pérez-Suárez G, Palacios F, Boursot P: Speciation and paraphylogeny in Western Mediterranean hares (Lepus castroviejoi, L. europaeus, $L$. granatensis and $L$. capensis) revealed by mitochondrial DNA phylogeny. Biochem Genet 1994, 32:423-436.

34. Guillaume GP, Heulin B, Arrayago MJ, Bea A, Brana F: Refuge areas and suture zones in the Pyrenean and Cantabrian regions: geographic variation of the female MPI sex-linked alleles among oviparous populations of the lizard Lacerta (Zootoca) vivipara. Ecography 2000, 23:3-10.

35. Steinfartz $S$, Veith $M$, Tautz $D$ : Mitochondrial sequence analysis of Salamandra taxa suggests old splits of major lineages and postglacial recolonizations of Central Europe from distinct source populations of Salamandra salamandra. Mol Ecol 2000, 9:397-410.

36. Rendell S, Ennos RA: Chloroplast DNA diversity in Calluna vulgaris (heather) populations in Europe. Mol Ecol 2002, I I:69-78.

37. García-París M, Alcobendas M, Buckley D, Wake DB: Dispersal of viviparity across contact zones in Iberian populations of fire salamanders (Salamandra) inferred from discordance of genetic and morphological traits. Evolution 2003, 57:129-143.

38. Konnert $M$, Bergmann $F$ : The geographical distribution of genetic variation in silver fir (Abies alba, Pinaceae) in relation to its migration history. Plant Syst Evol 1995, 196:19-30.

39. Puente Al, Altonaga K, Prieto CE, Rallo A: Delimitation of Biogeographical Areas in the Iberian Peninsula on the Basis of Helicoidea Species (Pulmonata: Stylommatophora). Glob Ecol Biogeogr Lett 1998, 7:97-II 3.

40. Fourcade J: Trois cent ans d'histoire au Pays basque (Urrugne, Socoa, Béhobie, Hendayes, Biriatou). 1901 Editions, France 1967.

41. Young A, Boyle T, Brown T: The population genetic consequences of habitat fragmentation for plants. Trends Ecol Evol 1996, II:413-418.

42. Mishalski S, Durka W: High selfing and high inbreeding depression in peripherl populations of Juncus atratus. Mol Ecol 2007, 16(22):4715-4727.

43. Pinho C, Harris DJ, Ferrand N: Contrasting patterns of population subdivision and historical demography in three western Mediterranean lizard species inferred from mitochondrial DNA variation. Mol Ecol 2007, I6: | | $91-1205$.

44. Willis KJ: The full-glacial forests of Central and Southeastern Europe. Quat Res 2000, 53:203-213

45. Kutzbach JF, Guetter PJ: The influence of changing orbital parameters and surface boundary conditions on climate simulations for the past $\mathbf{1 8 , 0 0 0}$ years. I Atmosph Sci 1986 43:1726-1759

46. Belles $X$ : Fauna cavernicola $i$ intersticial de la Peninsula lbèrica i les Illes Balears. In Monografie scientifiques, 4 Consell Superior d'Investigacions Cientifiques \& Editorial Moll, Mallorca; 1987.

47. Ferris C, Oliver RP, Davy AJ, Hewitt GM: Using chloroplast DNA to trace postglacial migration routes of oaks into Britain. Mol Ecol 1995, 4:73|-738.

48. Demesure $B$, Mush J: L'évolution de la forêt française après la dernière glaciation: l'apport de la palynologie, l'archéologie et de la biologie moléculaire. Les Dossiers de l'Environnement de I'INRA 200I, 2I:23-28.

49. Thomaz D, Guiller A, Clarke B: Extreme divergence of mitochondrial DNA within species of pulmonate land snails. Proc $R$ Soc Lond B 1996, 263:363-368.

50. Folmer O, Black M, Hoeh W, Lutz R, Vrijenhoek R: DNA primers for amplification of mitochondrial cytochrome $c$ oxidase subunit I from diverse metazoan invertebrates. Mol Mar Biol Biotechnol 1994, 3:294-299.

5I. Gittenberger E, Piel WH, Groenenberg DSJ: The Pleistocene glaciations and the evolutionary history of the polytypic snail species Arianta arbustorum (Gastropoda, Pulmonata, Helicidae). Mol Phyl Evol 2004, 30:64-73.

52. Thompson JD, Higgins DG, Gibson TJ: CLUSTAL W: improving the sensitivity of progressive multiple sequence alignment through sequence weighting, position specific gap penalties and weight matrix choice. Nucleic Acids Res 1994, 22:4673-4680.

53. Hall TA: BIOEDIT: a user friendly biological sequence alignment editor and analysis program for Windows 95/98/NT. Nucleic Acids Symp Ser 1999, 41:95-98.

54. Rozas J, Sánchez-DelBarrio JC, Messeguer X, Rozas R: DnaSP, DNA polymorphism analyses by the coalescent and other methods. Bioinformatics 2003, 19:2496-2497.

55. Schneider S, Roessli D, Excofier L: Arlequin, Version 2.0: a software for population genetic data analysis Genetics and Biometry Laboratory, University of Geneva, Geneva; 2000.

56. Swofford DL: PAUP* 4.0b Sinauer Associates, Sunderland, MA; 2002.

57. Huelsenbeck JP, Ronquist F: MRBAYES: Bayesian inference of phylogenetic trees. Bioinformatics 2000, I7(8):754-755.

58. Akaike $\mathrm{H}$ : Information theory and an extension of the maximum likelihood principle. Second International Symposium on Information Theory 1973:267-28I. [Akademiai Kiado, Budapest]

59. Nylander JAA, Ronquist F, Huelsenbeck JP, Nieves-Aldrey JL: Bayesian phylogenetic analysis of combined data. Syst Biol 2004, 53:47-67.

60. Felsenstein J: Evolutionary trees from DNA sequences: a maximum likelihood approach. J Mol Evol 198I, I7:368-376.

6I. Hasegawa M, lida Y, Yano T, Takaiwa F, Iwabuchi M: Phylogenetic relationships among eukaryotic kingdoms inferred from ribosomal RNA sequences. J Mol Evol 1985, 22:32-38.

62. Guindon S, Gascuel O: A simple, fast, and accurate algorithm to estimate large phylogenies by maximum likelihood. Syst Biol 2003, 52:696-704.

63. Posada D, Crandall KA: Modeltest: testing the model of DNA substitution. Bioinformatics 1998, 14:817-818.

64. Bandelt H-J, Forster P, Röhl A: Median-joining networks for inferring intraspecific phylogenies. Mol Biol Evol 1999, 16:37-48.

65. Fu YX: Statistical tests of neutrality of mutations against population growth, hitchhiking and background selection. Genetics 1997, 147:915-925.

66. Ramos-Onsins SE, Rozas J: Statistical properties of new neutrality tests against population growth. Mol Biol Evol 2002, 19:2092-2100.

67. Slatkin M, Hudson RR: Pairwise comparisons of mitochondrial DNA sequences in stable and exponentially growing populations. Genetics 1991, 129:555-562.

68. Rogers AR, Harpending $\mathrm{H}$ : Population-growth makes waves in the distribution of pairwise genetic-differences. Mol Biol Evol 1992, 9:552-569.

69. Drummond AJ, Rambaut A: BEAST, version 1.3 University of Oxford, Oxford, UK; 2005.

70. Johnson SG: Age, phylogeography and population structure of the microendemic banded spring snail, Mexipyrgus churinceanus. Mol Ecol 2005, 14:2299-23।I.

7I. Ozawa R, Okamoto K: Integrated palaeontological and molecular phylogenetic approaches to the study of phylogeny: a case study of Umbonium (Gastropoda). Chikyu Month 1993, 1 5:589-595. (In Japanese)

72. Rumbak E, Reid DG, Thomas RH: Reconstruction of phylogeny of I I species of Littorina (Gastropoda: Littorinidae) using mitochondrial DNA sequence data. The Nautilus Suppl 1994, 2:91-97.

73. Chiba S: Accelerated evolution of land snails Mandarina in the oceanic Bonin Islands: evidence from mitochondrial DNA sequences. Evolution 1999, 53:460-47|.

74. Marko PB: Fossil calibration of molecular clocks and the divergence times of geminate species pairs separated by the Isthmus of Panama. Mol Biol Evol 2002, I 9(I I):2005-202I.

75. The Museum of Natural History Elona quimperiana (Férussac 182I) [http://www.mnhn.fr/] 